\title{
The impact of the operation mode of CRACs on the temperature distribution of hot and cold aisles for the data center room
}

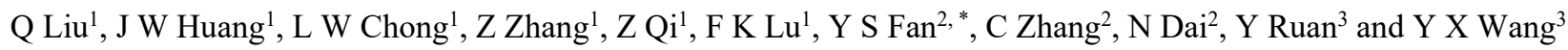 \\ ${ }^{1}$ National Computer Network Emergency Response Technical Team \& Coordination Center, 71780005-6, China \\ ${ }^{2}$ School of Environmental Science and Engineering, Tianjin University, Tianjin, China \\ ${ }^{3}$ Gongxintong (Beijing) Technology Co., Ltd, Beijing, China
}

\begin{abstract}
Redundancy is generally considered in the computer room air conditioners (CRACs) of the data center room. The position of the CRAC in the on state and the operating air volume have a great influence on the temperature distribution. The temperature distribution of the data center room will affect the failure rate of the servers and the energy consumption of the air-conditioning system. Taking a typical data center room as an example, this paper uses numerical simulation to study the influence of the on/off state and air volume of CRACs on the temperature distribution of the hot and cold aisles and energy consumption. The calculation results show that we should preferably turn off the staggered CRACs on the opposite side and close to the middle position under the partial load. When the IT load is unevenly distributed, it is irrational to use the setting method of even distribution of the air volume of each CRAC. The air volume of each CRAC should be appropriately adjusted based on the distribution characteristic of IT load to reduce the energy consumption.
\end{abstract}

\section{Introduction}

In recent years, with the development of internet and cloud computing, the number and scale of data center in many countries are expanding rapidly. At the same time, the high energy consumption of data center has attractted public attention. The energy consumption of data center is mainly composed of four parts: IT equipment, airconditioning system, power system and lighting system. Among them, the energy consumption of the airconditioning system accounts for about $40 \%$ [1].

Reducing the energy consumption of the airconditioning system can be achieved by rationally planning the airflow organization of the terminals [2]. Zhang [3] stated that a rational layout of the ventilation wall could greatly improve the distribution of airflow and the thermal environment of the data center room, so that the heat generated by the servers could be discharged outside in time, and the energy consumption of the airconditioning system could be reduced. Gao et al [4] stated that installing clapboard on the top of the rack on the cold aisle and partially enclosing the cold aisles to improve the airflow organization of the data center room could effectively reduce the mixing degree of hot and cold airflow, the waste of cooling, and the energy consumption of the air-conditioning system. Increasing the temperature of the data center room could also reduce the energy consumption of the air-conditioning system [5]. Taking the currently most widely used water-cooled chilled water system as an example, Dai et al [6] analyzed the energysaving potential and technology of the air-conditioning system of data center, and proposed the energy-saving operation strategies of increasing the chilled water supply temperature and the supply air temperature. The current research on reducing the energy consumption of the airconditioning system of data center mainly focuses on the refrigeration system and airflow organization, but the research on the operation mode of computer room air conditioner (CRAC) and the internal temperature distribution of the data center room is not in-depth.

Temperature had a significant impact on the failure rates of the servers of data center [7]. According to the ASHRAE Technical Committee (TC) 9.9 research report, the servers of data center have different failure rates in different temperature ranges.

As the temperature rose, the failure rate of the server would gradually increase [8]. Therefore, when setting the temperature inside the data center room, the failure rate of the server needs to be considered. In order to ensure the normal operation of the IT equipment in the data center, strict control of the temperature inside the data center room is required. Data Center Design Code (GB 501742017) limited the temperature of the cold aisle to be between $18{ }^{\circ} \mathrm{C}$ and $27{ }^{\circ} \mathrm{C}$ [9]. The temperature distributions of the hot and cold aisles affect the failure rate of the server and the energy consumption of the CRACs. Under the case of uneven temperature distribution, in order to avoid the phenomenon of excessively high temperature in local areas, the temperature in certain areas must be low. This uneven temperature distribution will increase the energy consumption of the air-conditioning system.

This paper uses 6 SigmaRoom software to establish a 3D model of the data center room [10], and simulates the temperature field and airflow field inside the room under 
different cases. We compare and analyze the temperature difference and airflow difference under different cases to study the impact of the operating number and parameter settings of CRAC on the temperature distributions of the hot and cold aisles and find a relatively rational operation strategy to guide the practice and reduce the energy consumption of the air-conditioning system.

\section{Model establishment and parameter setting}

\subsection{Model establishment}

In this paper, a data center room in Beijing, China is selected as the research object, and 6SigmaRoom software is used for simulation to analysis the energy consumption. Figure 1 shows the simulation model.

The typical precision data center room has an area of about $370 \mathrm{~m}^{2}$, with the total height of $5.9 \mathrm{~m}$, and the height of the raised floor is $1 \mathrm{~m}$. There are 118 server racks in the data center room, which are arranged in 6 rows (row A-F). The design IT load of each rack is $7 \mathrm{~kW}$, so the design IT cooling load of row $\mathrm{B}$ and $\mathrm{E}$ is $119 \mathrm{~kW}$, and the design IT cooling load of row A, C, D, and F is $147 \mathrm{~kW}$. The data center room is equipped with 8 CRACs, 6 for use and 2 for standby. Each CRAC has a rated air volume of 38000 $\mathrm{m}^{3} / \mathrm{h}$ and a rated sensible cooling capacity of $130.2 \mathrm{~kW}$. The air supply mode is underfloor air supply, and the cold aisles are closed whose floor grille has the hole fraction of $50 \%$.

Table 1. The operating states of CRACs under even IT load distribution.

\begin{tabular}{ccccccccc}
\hline Cases & CRAC01 & CRAC02 & CRAC03 & CRAC04 & CRAC05 & CRAC06 & CRAC07 & CRAC08 \\
\hline 1 & 1 & 1 & 1 & 0 & 0 & 1 & 1 & 1 \\
2 & 1 & 1 & 0 & 0 & 1 & 1 & 1 & 1 \\
3 & 1 & 0 & 1 & 1 & 1 & 1 & 0 & 1 \\
4 & 1 & 0 & 1 & 1 & 1 & 0 & 1 & 1 \\
5 & 1 & 0 & 1 & 0 & 1 & 1 & 1 & 1 \\
6 & 1 & 0 & 0 & 1 & 1 & 1 & 1 & 1 \\
7 & 0 & 1 & 1 & 1 & 0 & 1 & 1 & 1 \\
8 & 0 & 1 & 1 & 0 & 1 & 1 & 1 & 1 \\
\hline
\end{tabular}

Notes: ' 1 ' is on, ' 0 ' is off

For the second type of case, the set-point of the return air temperature of the CRACs is $27{ }^{\circ} \mathrm{C}$. CRAC02 and CRAC07 are turned off, while other CRACs are kept on.

The total air supply volume of the data center room is $152000 \mathrm{~m}^{3} / \mathrm{h}$. Table 2 shows the specific parameter of CRACs.

Table 2. The air volume setting of CRACs under uneven IT load distribution (unit: $\mathrm{m}^{3} / \mathrm{h}$ )

\begin{tabular}{ccccccccc}
\hline Cases & CRAC01 & CRAC02 & CRAC03 & CRAC04 & CRAC05 & CRAC06 & CRAC07 & CRAC08 \\
\hline 9 & 38000 & 0 & 38000 & 38000 & 38000 & 38000 & 0 & 38000 \\
10 & 34200 & 0 & 34200 & 34200 & 34200 & 34200 & 0 & 34200 \\
11 & 32300 & 0 & 32300 & 32300 & 32300 & 32300 & 0 & 32300 \\
12 & 30400 & 0 & 30400 & 30400 & 30400 & 30400 & 0 & 30400 \\
13 & 28500 & 0 & 28500 & 28500 & 28500 & 28500 & 0 & 28500 \\
14 & 30400 & 0 & 32300 & 32300 & 30400 & 30400 & 0 & 32300 \\
15 & 28500 & 0 & 32300 & 32300 & 30400 & 30400 & 0 & 32300 \\
16 & 22800 & 0 & 32300 & 32300 & 30400 & 30400 & 0 & 34200 \\
17 & 15200 & 0 & 32300 & 32300 & 30400 & 30400 & 0 & 34200 \\
\hline
\end{tabular}

\subsection{Simulation cases}

This paper simulates two types of cases for partial load. The first type contains 8 cases where the IT load is evenly distributed, and the IT load ratio of the server racks is $50 \%$. uneveristributed. The IT load ratio of the server in row $\mathrm{A}, \mathrm{B}$ and $\mathrm{C}$ is $30 \%$, and that in row $\mathrm{D}, \mathrm{E}$ and $\mathrm{F}$ is the operation states of the CRACs under various cases are shown in Table 1. $70 \%$.
The second type contains 9 cases where the IT load is modes are remained the same. The total air supply volume of the data center room is $152000 \mathrm{~m}^{3} / \mathrm{h}$ and evenly
supplied by the CRACs in the on state. The operating

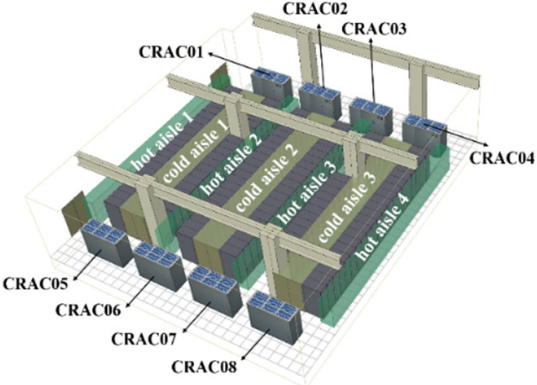

Figure 1. The 3D model of the typical precision data center room.

For the first type of case, the set-point of the return air 


\section{Results and discussion}

\subsection{Temperature field under even IT load distribution}

Figures 2 and 3 are the temperature statistics graphs of cold and hot aisles.

The number of CRAC in operation of cases 1 to 8 is 6 . According to the position of 2 CRACs turned off, 8 cases can be divided into 4 categories: the neighboring CRACs on the same side (case 2 and case 6), the non-neighboring CRACs on the same side (case 5 and case 8), the staggered CRACs on the different side (case 1 and case 3), the opposite CRACs on the different side (case 4 and case 7). As shown in Figures 2 and 3, when the number of CRAC turned on is the same, the temperature distribution of the hot and cold aisles is quite different, which indicates that turning on CRACs in different position has a great impact on the temperature distribution of the hot and cold aisles.

Two CRACs near hot aisle 1 are turned off in case 7, which causes CRAC02, CRAC03, CRAC06, and CRAC07 to supply lower temperature air under the fixed air volume to bear the load that should be borne by CRAC01 and CRAC05. This indirectly causes the air supply temperature of CRAC04 and CRAC08 to increase. Then the temperature of cold aisle 1 drops, while the temperature of cold aisle 3 rises, and the temperature of cold aisle 2 basically remains unchanged, as shown in Figure 2. The temperature of hot aisles 1 and 2 will drop, and the temperature of hot aisle 1 will drop more. Similarly, the temperature of hot aisles 3 and 4 will rise, and the rise of the hot aisle 4 will be greater, as shown in Figure 3.

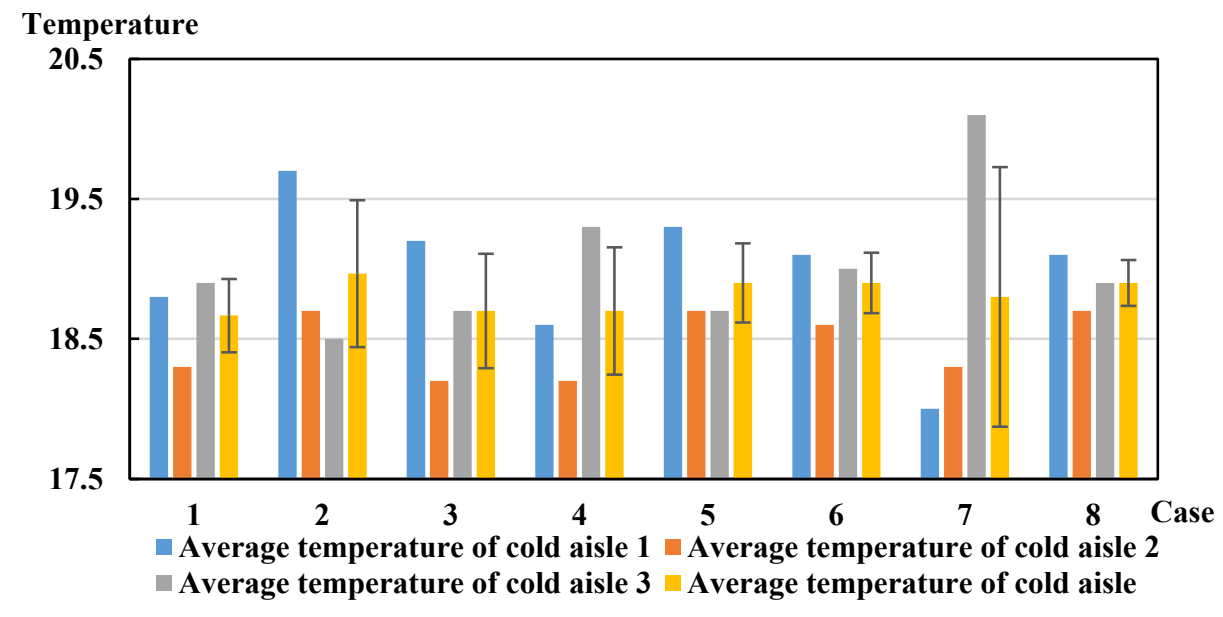

Figure 2. The temperature of cold aisle.

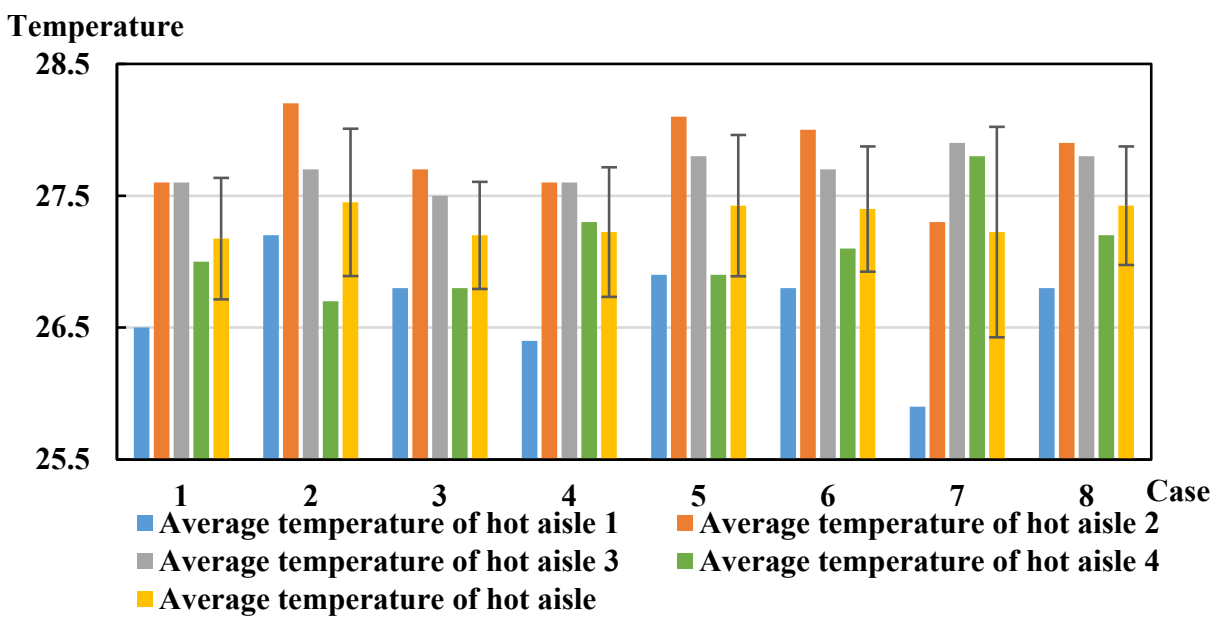

Figure 3. The temperature of hot aisle.

In case 4, the two CRACs close to the middle of the room are turned off, so their load is borne by the four CRACs relatively evenly, instead of being mainly borne by two of them as in case 7 . Therefore, the temperature field of the cold aisle of case 4 is more even than that of case 7 . In addition, the air in the hot aisle in case 4 is more easily mixed with the surrounding air, so the evenness of the temperature field distribution of the hot aisle in case 4 is better than that of case 7. Therefore, the distribution of the temperature field of the hot and cold aisles in case 4 is more even, as shown in Figures 4 and 5.

Figure 3 shows that the average temperature of hot aisles in cases 2, 5, 6, and 8 is higher. the common feature of these cases is that the two CRACs on the same side are turned off. This shows that it is irrational to turn off the two CRACs on the same side. Under the same temperature of hot aisle, these cases require more air volume, resulting in higher energy consumption. 
As shown in Figures 2 and 3, when the staggered CRACs on the different side are turned off, the indoor temperature field is more evenly distributed. The CRACs turned off in case 1 are at the edge, while the CRACs turned off in case 3 are in the middle, so the air in the hot aisle in case 3 is easier to be supplied from the surrounding CRACs. Therefore, the evenness of temperature of the hot aisle in case 3 is slightly better than that in case 1 .

From the above analysis, it can be concluded that under the premise of turning off two CRACs, case 3 is the most ideal choice. Considering the actual working conditions, when the load distribution in the data center room is relatively even, and the data center room is equipped with 8 CRACs ( 2 for standby), the remaining 6 of which are evenly distributed in the air supply volume, we conclude that we should first select the staggered position preferably close to the middle on the different side to arrange the standby CRACs.

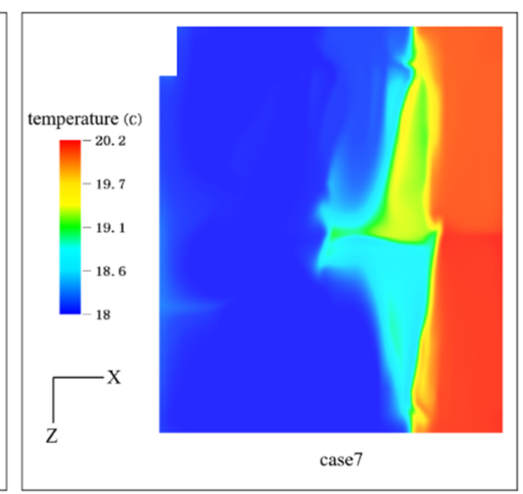

Figure 4. The temperature distribution at the height of $-0.4 \mathrm{~m}$ (case 4 and case 7).

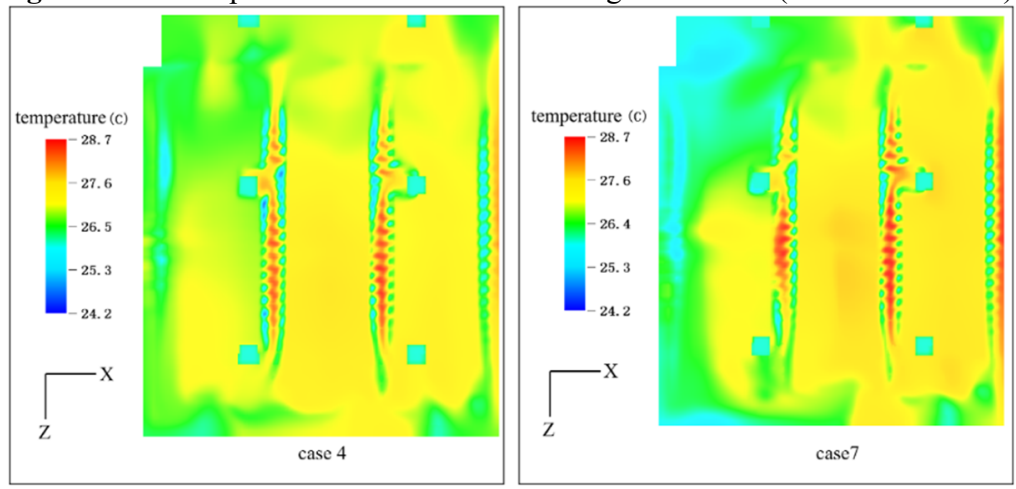

Figure 5. The temperature distribution at the height of $2.3 \mathrm{~m}$ (case 4 and case 7).

\subsection{Temperature field under uneven IT load distribution}

Figures 6 and 7 are the temperature statistics graphs of cold and hot aisle.

The total air supply volumes of the data center room in cases 9 to 13 are $228000 \mathrm{~m}^{3} / \mathrm{h}, 205200 \mathrm{~m}^{3} / \mathrm{h}, 193800$ $\mathrm{m}^{3} / \mathrm{h}, \quad 182400 \mathrm{~m}^{3} / \mathrm{h}$ and $171000 \mathrm{~m}^{3} / \mathrm{h}$ respectively. Compared with the rated air volume, the relative air volume ratio of each CRAC in cases 9 to 13 is $100 \%, 90 \%$, $85 \%, 80 \%$ and $75 \%$ respectively. The reduce of total air volume leads to the decrease of the air supply temperature, which leads to the decrease of the average temperature of the cold and hot aisles and the increase of the standard deviation of the temperature of the cold and hot aisles. However, Figure 6 shows that the average temperature of cold aisle 3 in case 12 is slightly lower than the lower limit $\left(18{ }^{\circ} \mathrm{C}\right)$ of the cold aisle's specified temperature by $0.1^{\circ} \mathrm{C}$, while the average temperature of cold aisle 3 in case 13 is already $0.8^{\circ} \mathrm{C}$ lower than $18{ }^{\circ} \mathrm{C}$, which indicates that the air supply volume of some CRACs is too small, causing the air supply temperature to be too low. The simulation results show that the air supply temperatures of CRAC04 and CRAC08 in case 13 are $17.4{ }^{\circ} \mathrm{C}$ and $16.8{ }^{\circ} \mathrm{C}$ respectively. As shown in Figure 8, the temperature of most areas of cold aisle 3 and some areas of cold aisle 2 has fallen below the limit value of $18^{\circ} \mathrm{C}$. The simulation result and the inference shows that under the condition of partial load and uneven IT load distribution, reducing the total air supply volume of the data center room within a certain range will have a certain impact on the temperature distribution of the hot and cold aisles, but it can still meet the requirement.

When the IT load is unevenly distributed, if the air volume of the CRACs in the on state is evenly distributed, the total air supply volume of the data center room should be greater than the total air supply volume determined by the air volume ratio equal to the load ratio to meet the requirement. As shown in Figure 6, under the premise that the air volume of the CRACs in the on state is evenly 
distributed, the total air supply volume of the data center room should be greater than $80 \%$ of the design air volume.

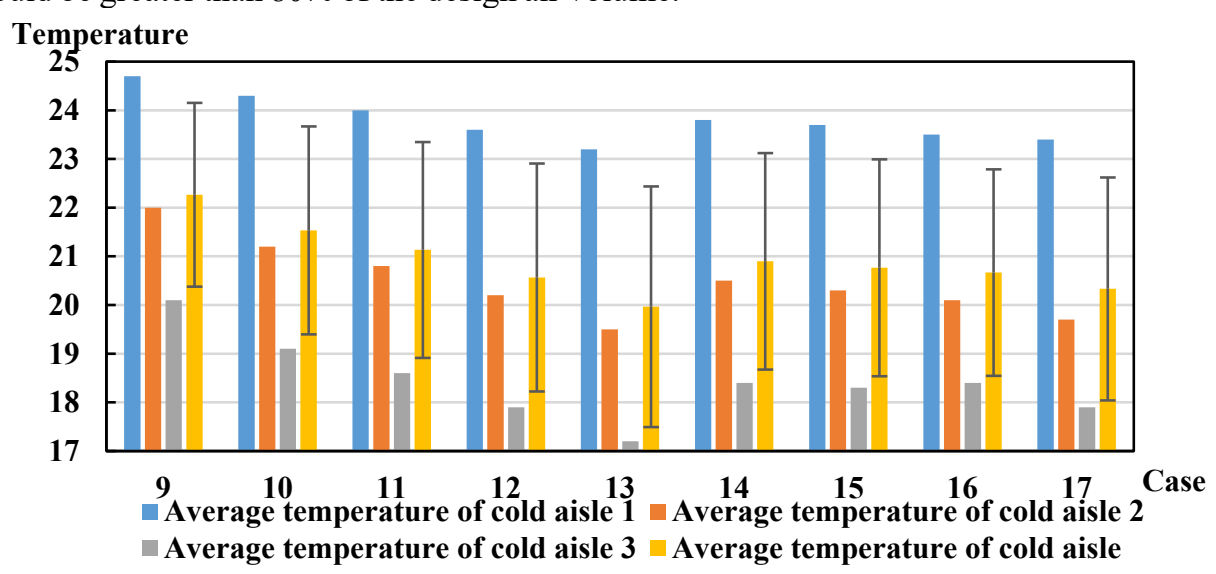

Figure 6. The temperature of cold aisle.

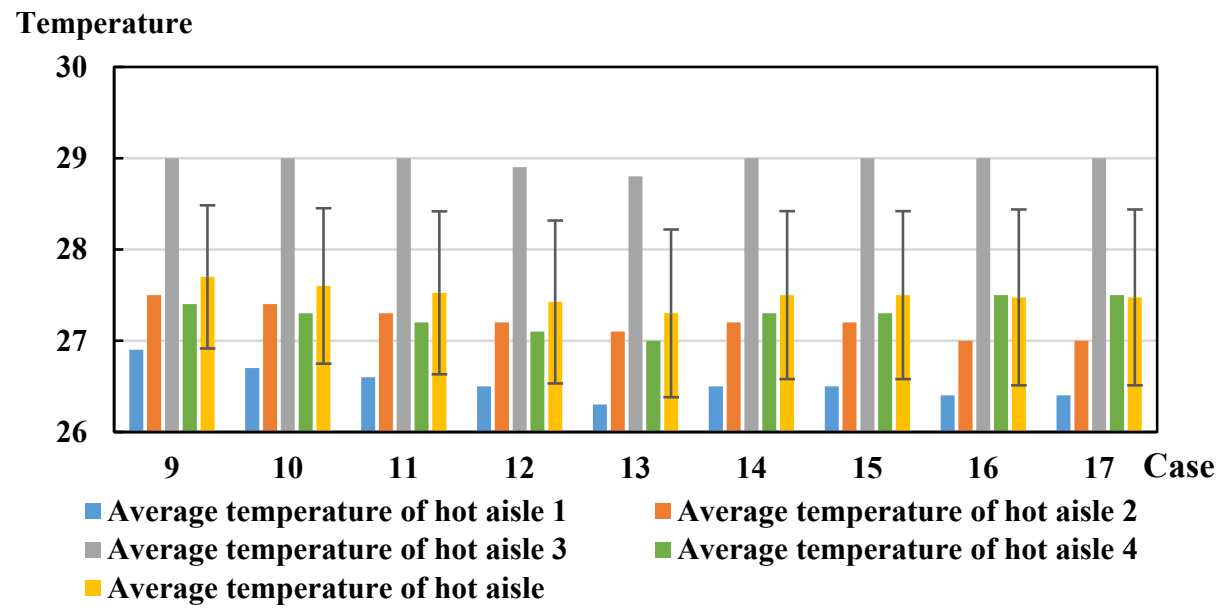

Figure 7. The temperature of hot aisle.

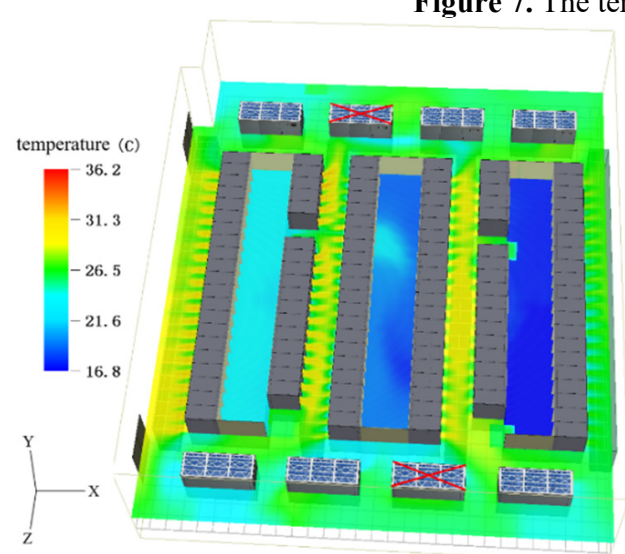

Figure 8. The temperature distribution of data center room (case 13).

When the air volume of the CRACs in the on state is evenly distributed, the total air supply volume of is higher, meanwhile the load of the fan is higher. If the air supply volume on the high IT load side is kept unchanged, the load of fan can be reduced by appropriately reducing the air supply volume on the low IT load side. The temperature of cold aisles in case 11 is within the specified range, and the air volume is small. Based on case 11, we keep the air volume of the CRACs on the high load side unchanged

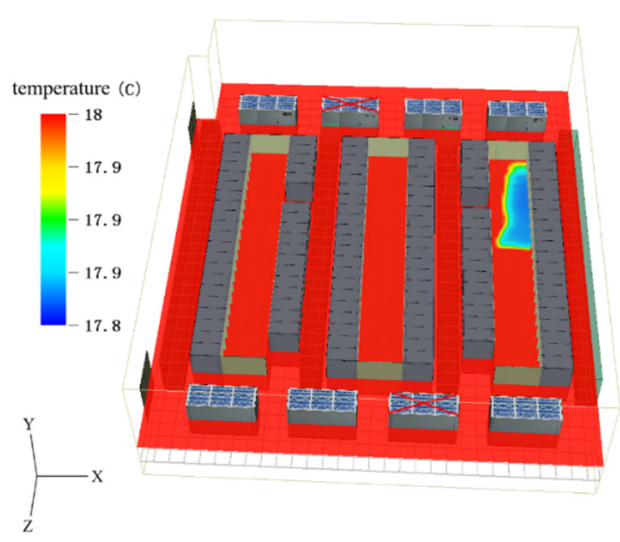

Figure 9. The temperature distribution of data center room (case 17).

and reduce the air volume of the CRACs on the low load side to obtain case 14 , and the total air supply volume in case 14 is $188100 \mathrm{~m}^{3} / \mathrm{h}$ (the relative air volume ratio is $82.5 \%$ ). It can be seen from Figures 6 and 7 that there is almost no difference in the temperature distribution of the cold and hot aisles between cases 11 and 14. Compared with case 11, the air volume of CRAC01, CRAC05, and CRAC06 in case 14 all reduces by $1900 \mathrm{~m}^{3} / \mathrm{h}$, and the load of fan has been reduced to a certain extent. 
Based on case 14, the air volume of CRAC01 in case 15 is continuously reduced. The total air supply volume is $186200 \mathrm{~m}^{3} / \mathrm{h}$ (the relative air volume ratio is $81.6 \%$ ). The air volume of CRAC08 is increased in case 16 on the basis of case 15, and the air volume of CRAC01 is continuously reduced. The total air supply volume is reduced to 182400 $\mathrm{m}^{3} / \mathrm{h}$ (the relative air volume ratio is $80 \%$ ). Figures 6 and 7 show that it still meets the requirements in cases 15 and 16. The air volume of CRAC01 is continuously reduced in case 17 on the basis of case 16, and the total air supply volume is reduced to $176700 \mathrm{~m}^{3} / \mathrm{h}$ (the relative air volume ratio is $77.5 \%$ ). The simulation results show that the air supply temperature of $\mathrm{CRAC} 08$ is $17.8{ }^{\circ} \mathrm{C}$, which is already lower than the limit value of $18{ }^{\circ} \mathrm{C}$, as shown in Figure 9. The results show that adjusting the air volume of each CRAC according to the IT load can reduce the energy consumption.

\section{Conclusions}

For safety, redundancy should be considered in CRACs of the data center room. When the position of the CRACs in the off state is different, the air distribution will be different. The simulation results show that we should avoid turning off the CRACs on the same side or close to the corner, that is, the standby CRACs should be preferentially arranged on the opposite side and preferably close to the middle.

In addition to the position of the CRAC, the air volume of each CRAC also has an impact on the air distribution and temperature distribution. Under the premise that the IT load in the data center room is evenly or relatively evenly distributed, the setting method of evenly distributed air volume can be adopted. While in the case of uneven distribution of IT load, it is irrational to adopt the setting method of even distribution of air volume. For a specific IT load distribution, the requirement can be met under a variety of combination of air supply temperature and air supply volume. But the greater the air volume, the higher the energy consumption of the fan. The simulation results show that the total air volume required by even air volume setting is the maximum. The air volume of each CRAC should be adjusted based on the distribution characteristic of IT load to reduce the energy consumption.

In practical engineering, the energy consumption of data center room can be reduced by optimizing the operation strategy of CRACs. This paper proposes the operation strategy according to the IT load, which can reduce the operation energy consumption of CRACs on the premise of meeting the temperature requirements of the data center room.

Future study can explore better operation strategy of CRACs under different load characteristics, and combine the air conditioning operation strategy and terminal regulation strategy to reduce the energy consumption of data center.

\section{Acknowledgments}

The 6SigmaRoom used in this paper was provided by Beijing Rainspur Technology Co., Ltd and Future
Facilities Ltd, which provided great help for the completion of the paper. On the occasion of the completion of the paper, I would like to extend my most heartfelt thanks and sincere respect to both companies.

Special thanks to Mr. Yang Chao from Beijing Rainspur Technology Co., Ltd for his hard work and efforts in helping us get and run the software.

\section{References}

1. Jiacheng $\mathrm{N}$ and Xuelian B 2017 A review of air conditioning energy performance in data centers Renew. Sustain. Energy Rev. 67 625-40

2. Emelie Wibron, Anna-Lena Ljung and T. Staffan Lundström 2018 Computational Fluid Dynamics Modeling and Validating Experiments of Airflow in a Data Center Energies 11

3. Qun Z 2021 Research on the influence of air distribution design on the cooling effect of data room Telecom. Eng. Techn. Stand. 34 16-21

4. Caifeng G, Zhen Y and Jianlin W 2013 Thermal environment analysis and air distribution optimization for a typical data room $J$. $H V \& A C \mathbf{4 3}$ 101-6

5. Yogesh Fulpagare and Atul Bhargav 2015 Advances in data center thermal management Renew. Sustain. Energy Rev. 43 981-96

6. Xinqiang D, Weike D, Jianru H, Zhongbin $\mathrm{Z}$ and Daoming Z 2020 Research on energy consumption and energy saving application of air conditioning system in data center China Plant Engineering 4 1214

7. Thiago Lara Vasques, Pedro Moura and Aníbal Almeida 2019 A review on energy efficiency and demand response with focus on small and medium data centers Energ. Effic. 12 1399-428

8. ASHRAE Technical Committee 2011 Thermal Guidelines for Data Processing EnvironmentsExpanded Data Center Classes and Usage Guidance.

9. Ministry of Housing and Urban-Rural Development of the People's Republic of China 2017 Data center design specification GB50174-2017

10. Peter V. Nielsen 2015 Fifty years of CFD for room air distribution Build. Environ. 91 78-90 